the socialist revolution? Vuorela has exemplified how close class and gender are intertwined. It is impossible to separate the one from the other, both have to be considered as points of every analysis. Vuorela makes that clear. Even though it might at some points of her analysis seem questionable whether all aspects of a specific culture can be subsumed under the concepts of MOP and MOHR, her work does not become reductionist in that sense. It is an excellent example for understanding the modes of production and human reproduction as one system and an appeal to consider gender as well as class.

Johanna Schoen

\title{
Eckart Putzier
}

\section{Die Ermächtigungen des Außenwirtschaftsgesetzes}

Stuttgart, Fachverlag für Wirtschaft und Steuern Schäffer GmbH \& Co., 1987, XXIII + 127 S., DM 42,-

Anlaß der ursprünglich als Freiburger Dissertation gefertigten Studie war wohl nicht zuletzt (s. S. V) ein Jubiläum - 25 Jahre seit Inkrafttreten der Erstfassungen von AWG (vom 28. 4. 1961, BGBl. I, 481) und der zu dessen Durchführung erlassenen AWV (vom 22. 8. 1961, BGBl. I, 1381), je zum 1. 9. 1961. Ähnlich wie bei einem ebenfalls vor kurzem gef eierten Jahrestag - 40 Jahre DM/Währungsreform (20.6. 1948) - muß aber auch hier gefragt werden, ob denn die schiere Dauerhaftigkeit einer gesetzlichen Regelung bereits ihren Erfolg belege. Wenn nun die Deutsche Mark zwar nicht unmittelbar Gefahr läuft, von einer europäischen Währung(seinheit) verdrängt zu werden, so ist doch die Entwicklung der nationalen außenwirtschaftsrechtlichen Vorschriften durch einen zunehmenden Aushöhlungsprozeß gekennzeichnet: Putzier dürfte allenfalls bezogen auf das AWG selbst mit der Feststellung recht haben (S. 12), die Regelung habe "nur unwesentlich an Bedeutung verloren". Im Hinblick auf den Waren-Verkehr zumindest hat sich die Funktion inner-/mitgliedstaatlichen Rechts weithin auf das Feld bloßer Anwendung gemeinschaftsrechtlicher Normen zurückgebildet, wie schon ein Blick auf die Einund Ausfuhrbestimmungen der jüngst neugefaßten AWV zeigt (BGBl. 1986 I, 2671). Der Autor verkennt dies keineswegs, zeichnet vielmehr die "Einschränkung der Anwendbarkeit des AWG durch EG-Recht" vorab allgemein (S. $10 \mathrm{ff}$.) und sodann bei den besonderen Ermächtigungstatbeständen (z.B. S. 54, 61, 83) nach. Aus der Themenstellung fast völlig heraus fällt (leider!) der heute - man denke nur an die Sujets der Uruguay-Runde des GATT - immer gewichtigere Bereich des auch hierzulande weithin spezialgesetzlich geregelten grenzüberschreitenden Dienstleistungsverkehrs, insonderheit im Finanzwesen. Das Buch befaßt sich lediglich mit den dafür maßgeblichen, aber nur eher periphere Probleme behandelnden $\S$ 15-21 AWG (S. 87 ff.), von deren Möglichkeiten immerhin kaum Gebrauch gemacht wurde. 
Die Arbeit besticht vor allem durch ihren exakten, fast schon pedantischen Aufbau. Einem kurzen historischen Rückblick folgt im 2. Kapitel (S. $5 \mathrm{ff}$.) eine nicht wesentlich längere Verortung des AWG im umfassenderen Außenwirtschafts-Recht nationalen, gemeinschaftlichen wie internationalen Ursprungs. Daran schließt sich eine differenzierte Erörterung der (generellen) Regelungstechnik des AWG an, beginnend beim verfassungsrechtlich fundierten Freiheitsgrundsatz des $§ 1$ I (S. 15) über den Rahmen-Charakter der gesetzlichen Regelung und dessen Ausfüllung durch Rechtsverordnungen - diese wiederum kompiliert in einer AWV (S. 22 ff.) - bis hin zur Genehmigungserteilung für einzelne Rechtsgeschäfte und Handlungen (S. 42 ff.). Bei der verfassungsrechtlichen Würdigung des Einspruchsrechts des Bundestages gegenüber Regierungs-Verordnungen (§ 27 II AWG) hätte Putzier noch auf die entsprechende Regelung in Art. 109 IV 4 GG, $\S 20$ V 2 StWG hinweisen können (S. 24). Die Darstellung zur Frage, ob und wann Eingriffe in bereits geschlossene Verträge zulässig sind ( $\$ 2$ II 3 AWG, s. S. 34 ff.), befaßt sich auch mit dem eigentums-/grundrechtlichen Aspekt; etwas verkürzend dürfte freilich das Argument gegen das Vorliegen einer Enteignung i.S.v. Art. 14 III GG sein, daß der Gesetzgeber dies ausweislich des Berichts des BT-Ausschusses 1961 anders gesehen habe (s. S. 38).

Im 4. Kapitel, welches vom Umfang her fast zwei Drittel der Arbeit einnimmt, wendet sich der Autor zunächst den allgemeinen ( $\S 5-7$ AWG; S. 47 ff.), sodann den besonderen Ermächtigungsnormen für Beschränkungen des Waren- ( $\S 8-14$; S. 64 ff.), Dienstleistungs- und Kapitalverkehrs ( $\S 6$ a, 22-24; S. 98 ff.) zu und schenkt abschließend auch den (intensiv genutzten) Möglichkeiten zum Erlaß von Verfahrens- und Meldevorschriften sein Augenmerk ( $\S 26,46$ III AWG; S. 109 ff.).

Die Ausführungen sind durchweg lesbar und lesenswert gehalten; Putzier scheut vor verfassungsrechtlicher Kritik nicht zurück, etwa in bezug auf die unzulängliche Zitierungspraxis unter Verstoß gegen Art. 80 I 3 GG (S. 50, 112), aber auch im Hinblick auf die fehlende Vereinbarkeit des $\S 17$ AWG mit Zensurverbot und Kunstfreiheitsgewährleistung (Art. 5 I 3, III GG; s. S. 91). In einigen Punkten freilich wäre etwas wagemutigeres Ausbrechen aus dem Gatter festgefügter Auffassungen - Putzier stützt sich weithin auf die Kommentare zum AWG, die fast alle nicht neuesten Datums sind - durchaus ein Gewinn gewesen, weil sich alte Begründungen nicht mehr ohne weiteres aufrechterhalten lassen. So mag heute zweif elhaft sein, ob zwischenstaatliche "Verpflichtungen" i.S.v. § 5 AWG allein aus "hard law" resultieren können, wie Putzier of fenbar annimmt (S. 48). Welche sekundären Rechtsakte von EG-Organen dieser Vorschrift überhaupt noch unterfallen (und nicht schon dem § 1 II AWG), nachdem Richtlinien sehr wohl auch (vertikale, wenn nicht gar horizontale) Wirkungen in Mitgliedstaaten zeitigen (können), bleibt in der Schwebe (s. S. 47, 49). Eine Abgrenzung zwischen Retorsion und Repressalie bei der Anwendung des § 6 I AWG unterbleibt (S. 53) - was schreibt also nationales Recht beim Gebrauch letzteren Instruments vor? Der gerade für Entwicklungsländer einschlägige Anwendungsfall des $\S 44$ a AWV (in der 1983er Fassung) - Genehmigungspflicht für Verträge zwischen gebietsansässigen Prüfungsgesellschaften und Gebietsfremden 
über die ständige Prüfung der Preise von Waren und Dienstleistungen, die für fremde Wirtschaftsgebiete bestimmt sind - wird lediglich notiert, aber nicht weiter kommentiert (S. 55); dabei wurden hierzu in den vergangenen Jahren diverse Aufsätze publiziert (vgl. RIW 1979, 809; 1980, 88; 1982, 14; 1984, 353; 1985, 116). Bei der Darstellung der Abwehr schädigender Einwirkungen auf die Bundesrepublik macht Putzier deutlich, daß mittels § 6 II AWG auch auf konträre Politik und/oder Ideologie reagiert werden könnte (S. 56); wenn daher hiervon bislang nur sozialistische Staaten ("Zentralverwaltungswirtschaften () betroffen wurden, so muß dies mithin nicht immer so bleiben! Die Funktion des Außenwirtschaftsverkehrs(rechts) als Mittel und Waffe der Außenpolitik (S. 119; dazu auch Friedrich, NJW 1980, 2620) zeigt sich ferner, wie endlich im Kapitel über die "Lenkungskonzeption des AWG" nochmals hervorgehoben wird, in den Befugnissen mit allgemeinpolitischer Zielsetzung, vor allem in $\S 7$. Gerade dabei lassen der EWG-Vertrag (insbes. Art. 36, 223 und 224) sowie GATT (Art. XXI) stets noch hinreichend Raum für ein autonomes Vorgehen ihrer staatlichen Mitglieder (S. 58, 61 f.). Putzier nennt als Anwendungsfälle die inzwischen der Vergangenheit angehörenden Sanktionen gegen Süd-Rhodesien/Zimbabwe und den Iran sowie die Embargoliste des COCOM, die über $\S 5 \mathrm{AWV}$ in das nationale (deutsche) Recht einbezogen wird (S. 62 f.). Nicht zuletzt an diesem Vorgang hätte sich freilich präziser aufzeigen lassen, wie auch unverbindliche Rechtsakte internationaler Provenienz innerstaatliche Rechtsetzung veranlassen können, wenngleich hier ein legislatives Untätigbleiben nicht notwendig gegen Völker(vertrags)recht verstößt. Daß kein Wort zu Südafrika fällt, ist nicht dem Autor anzukreiden, sondern der Zurückhaltung der hiesigen Außen(wirtschafts)politik geschuldet.

Solche Detailkritik soll nicht das Gesamturteil über ein gelungenes Werk schmälern, vielmehr auf die Notwendigkeit hindeuten, die von Putzier in begrenztem Rahmen beackerte Thematik in weiteren Bereichen juristisch zu durchdringen. Daß die freiheitliche Konzeption des AWG nicht bloße Proklamation ist, vielmehr gerade beim Vergleich auf andernorts (bzw. früher auch in Deutschland) vorhandene und praktizierte Eingriffskompetenzen sich of fenbart, bleibt nämlich nicht unerwähnt (S. $114 \mathrm{f}$.). Die Forderung nach Abbau protektionistischer Normen als einem Mittel zur Bewältigung der internationalen Verschuldungsprobleme trifft so den bundesdeutschen Gesetzgeber nur in geringem Maße. Die Bundesregierung hingegen sollte nicht zuletzt bei ihrer Mitwirkung im Rat der EG nach Möglichkeit für freien Außenwirtschaftsverkehr eintreten, um der auch ihr auferlegten Verpflichtung, Grundrechte zu achten und zu schützen (Art. 1 I 2, II GG), nicht allein im nationalen Bereich und für die eigenen Staatsangehörigen nachzukommen.

Ludwig Gramlich 